Pure Appl. Chem., Vol. 76, No. 9, pp. 1605-1619, 2004.

(C) 2004 IUPAC

\title{
Palladium-catalyzed arylation of linear and cyclic polyamines*
}

\author{
Irina P. Beletskaya ${ }^{\ddagger}$ and Alexey D. Averin \\ Department of Chemistry, Lomonosov Moscow State University, Leninskie Gory, \\ Moscow 119992, Russia
}

\begin{abstract}
Synthetic protocols for the palladium-catalyzed arylation of various linear and cyclic polyamines and polyoxapolyamines has been worked out. $\mathrm{Pd}(0)$ and $\mathrm{Pd}(\mathrm{II})$ complexes with such phosphine ligands as dppf, BINAP, PPF-OMe, $\mathrm{P}(t \mathrm{Bu})_{3}$, 2-ditert-butylphosphino$1,1^{\prime}$-biphenyl have been explored in the catalytic amination reactions. Monoamination of chloro-, bromo-, and iodoarenes with di-, tri-, and tetraamines have been carried out, conditions for di- and polyarylation of linear polyamines have been elaborated. Successful arylation of 1,4,7,10-tetraazacyclododecane (cyclene) and 1,4,8,11-tetraazacyclotetradecane (cyclam) have been conducted. Intramolecular diamination of dihaloarenes such as 1,2-dibromobenzene, 2,6-dichlorobromobenzene, 1,3-dibromobenzene, 1,8-dichloroanthracene, 1,8-dichloroanthraquinone, 1,5-dichloroanthracene, and 1,5-dichloroanthraquinone afforded corresponding polyazamacrocycles containing arene moieties. For the first time, a convenient one-pot synthesis of the face-to-face arranged bismacrocyclic systems has been carried out.
\end{abstract}

\section{INTRODUCTION}

Polyamines attract the constant interest of chemists due to their wide use in pharmacology [1], coordination chemistry [2,3], and in the research of supramolecular systems [4]. Alkyl- and benzyl-substituted linear polyamines have been used as polydentate ligands for complexing palladium [5], copper [6], and other metals [7], and as fluorescence sensors for detecting a variety of metals such as $\mathrm{Ni}, \mathrm{Zn}, \mathrm{Cd}$, and $\mathrm{Pb}$ [8]. As a result, numerous convenient approaches to $N$-alkyl- and $N$-benzyl-substituted polyamines have been proposed. We believe that direct linking of arene moieties to the nitrogen atom of polyamines will enhance interaction between the polyamine chain and the arene group with appropriate photochemical properties. This will increase the sensing properties of such molecules.

Noncatalytic methods for the synthesis of arylamines often have such drawbacks as harsh conditions, strong dependence on the substituents in the arene fragment, and necessity of protection-deprotection steps. This strictly limits the synthesis of arylated polyamines, especially nonsymmetric derivatives. Multistep syntheses were often required to prepare even simple species [9]. An indirect method comprising several steps was used for the preparation of the $N$-aryl-1,2-ethanediamines [10]. A real breakthrough in the amination of aryl halides using palladium catalysis was achieved in the late 1990s after innovative works conducted by the groups of Buchwald and Hartwig [11]. It became the most important technique due to its obvious advantages, i.e., a wide choice of amines and substrates with various substituents, high yields, and, in many cases, mild condition protocols.

\footnotetext{
*Plenary lecture presented at the XVII Mendeleev Congress on General and Applied Chemistry, Kazan, Tatarstan, Russia, 21-26 September 2003. Other presentations are published in this issue, pp. 1605-1798.

¥Corresponding author: E-mail: beletska@org.chem.msu.ru
} 


\section{SYNTHESIS OF LINEAR ARYLATED POLYAMINES}

\section{Monoarylation of di- and polyamines}

We have thoroughly investigated the possibilities of the palladium-catalyzed arylation of polyamines for the synthesis of mono- and polyarylated species [12]. At first, the amination of various aryl bromides $\mathbf{2} \mathbf{a}-\mathbf{h}, \mathbf{k}-\mathbf{m}$ and aryl iodides $\mathbf{2} \mathbf{i}, \mathbf{j}$ with a model 1,3-diaminopropane 1a was carried out (Scheme 1).

\section{Scheme 1}

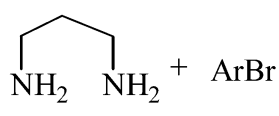

1a

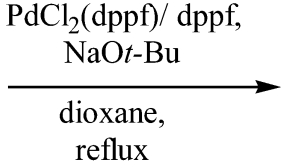<smiles>NCCCN</smiles>

3-13

The reaction employed $0.5-2 \mathrm{~mol} \%$ of $\mathrm{PdCl}_{2} / \mathrm{dppf}$ complex with a twofold excess of a free dppf ligand. Three equivalents of the diamine were taken to suppress its diarylation. Two equivalents of sodium tert-butoxide were used, and the reaction was run until completion in each case. The data obtained are collected in Table 1. Compared to bromobenzene, $p$-bromobiphenyl appeared to be more active, introduction of donor substituents in the benzene ring hindered the reaction and lowered the yields, electron-withdrawing benzoyl and nitrile groups afforded an excellent yield $\left[\mathrm{Pd}(\mathrm{dba})_{2}\right.$ was used instead of $\mathrm{PdCl}_{2}$ (dppf) complex in the last case], but some other electron-deficient groups proved to be sensitive to sodium tert-butoxide and this led to lower yields. The best results were achieved with active 1-bromonaphthalene and 9-bromoanthracene. Aryl iodides were not found to be advantageous to corresponding aryl bromides as they provided similar yields of arylated diamines.

Table 1 Arylation of 1,3-diaminopropane $1 \mathbf{a}$.

\begin{tabular}{lcc}
\hline Aryl bromide & $\mathrm{PdCl}_{2}(\mathrm{dppf}) / \mathrm{dppf}$, mol\% & Product (yield, \%) \\
\hline $\mathrm{C}_{6} \mathrm{H}_{5} \mathrm{Br} \mathbf{2 a}$ & $1 / 2$ & $\mathbf{3}(60)$ \\
$p-\mathrm{MeC}_{6} \mathrm{H}_{4} \mathrm{Br} \mathbf{2 b}$ & $1 / 2$ & $\mathbf{4}(51)$ \\
$p-\mathrm{C}_{6} \mathrm{H}_{5} \mathrm{C}_{6} \mathrm{H}_{4} \mathrm{Br} \mathbf{2 c}$ & $0.5 / 1$ & $\mathbf{5}(75)$ \\
$p-\mathrm{MeOC}_{6} \mathrm{H}_{4} \mathrm{Br} \mathbf{2 d}$ & $5 / 10$ & $\mathbf{6}(19)$ \\
$o-\mathrm{MeOC}_{6} \mathrm{H}_{4} \mathrm{Br} \mathbf{2 e}$ & $1.5 / 3$ & $\mathbf{7}(41)$ \\
$p-\mathrm{CF}_{3} \mathrm{C}_{6} \mathrm{H}_{4} \mathrm{Br} \mathbf{2 f}$ & $2 / 4$ & $\mathbf{8}(53)$ \\
$\left.p-\mathrm{MeC}_{2} \mathrm{O}\right) \mathrm{C}_{6} \mathrm{H}_{4} \mathrm{Br} \mathbf{2 g}$ & $0.5 / 1$ & $\mathbf{9}(21)$ \\
$p-\mathrm{NCC}_{6} \mathrm{H}_{4} \mathrm{Br} \mathbf{2 h}$ & $4 / 6$ & $\mathbf{1 0}(95)$ \\
$\mathrm{C}_{6} \mathrm{H}_{5} \mathrm{I} \mathbf{2 i}$ & $1 / 2$ & $\mathbf{3}(68)$ \\
$p-\mathrm{MeC}_{6} \mathrm{H}_{4} \mathrm{I} \mathbf{2 j}$ & $0.5 / 1$ & $\mathbf{4}(49)$ \\
$p-\mathrm{ClC}_{6} \mathrm{H}_{4} \mathrm{C}(\mathrm{O}) \mathrm{C}_{6} \mathrm{H}_{4} \mathrm{Br} \mathbf{2 k}$ & $1 / 2$ & $\mathbf{1 1}(77)$ \\
1-Bromonaphthalene 2l & $0.5 / 1$ & $\mathbf{1 2}(89)$ \\
9-Bromoanthracene 2m & $1 / 2$ & $\mathbf{1 3}(85)$ \\
\hline
\end{tabular}

We investigated a model $p$-bromobiphenyl $\mathbf{2 c}$ in the reactions with different polyamines. Reaction conditions mentioned above were applied in this process (Scheme 2). 
<smiles>[R]N(C([Y])(C)C(C)(C)C(C)(C)N)C([Y])(CC(C)(C)N)C(C)(C)N</smiles><smiles>[Y]C(CC(C)(C)Nc1ccc(-c2ccccc2)cc1)N([R])C([Y])(C)C(C)(C)CC(C)(C)N</smiles>

2c

$\mathrm{k}=0,1 ; 1=0,1 ; \mathrm{x}=0,1,2$

$\mathrm{R}=\mathrm{H}, \mathrm{Me}$

\section{Scheme 2}

Corresponding arylamines 14-20 were obtained in high yields, selective arylation of primary amino groups in the presence of secondary amino groups was observed in all cases (Table 2). If the diamines branched at $\beta$-carbon atom are used, this does not affect the yield of the product. Branching at $\alpha$-carbon atom results in lower yields, less regioselectivity of the process in the case of $p$-bromobiphenyl. When employing more active 1-bromonaphthalene, the reaction becomes more regioselective (Table 3, Scheme 3).

Table 2 Amination of $p$-bromobiphenyl.

\begin{tabular}{|c|c|c|}
\hline Amine & $\begin{array}{c}\mathrm{PdCl}_{2}(\mathrm{dppf}) / \mathrm{dppf} \\
\mathrm{mol} \%\end{array}$ & $\begin{array}{l}\text { Product } \\
\text { (yield, \%) }\end{array}$ \\
\hline $\mathrm{NH}_{2} \mathrm{CH}_{2} \mathrm{CH}_{2} \mathrm{NH}_{2} \mathbf{1 b}$ & $1 / 2$ & $14(64)$ \\
\hline $\mathrm{NH}_{2} \mathrm{CH}_{2} \mathrm{CH}_{2} \mathrm{NHCH}_{2} \mathrm{CH}_{2} \mathrm{NH}_{2} \mathbf{~} \mathbf{c}$ & $1 / 2$ & $15(65)$ \\
\hline $\mathrm{NH}_{2} \mathrm{CH}_{2} \mathrm{CH}_{2} \mathrm{CH}_{2} \mathrm{NHCH}_{2} \mathrm{CH}_{2} \mathrm{CH}_{2} \mathrm{NH}_{2} \mathbf{1 d}$ & $1 / 2$ & $16(77)$ \\
\hline $\mathrm{NH}_{2} \mathrm{CH}_{2} \mathrm{CH}_{2} \mathrm{CH}_{2} \mathrm{NHCH}_{2} \mathrm{CH}_{2} \mathrm{NH}_{2} \mathbf{1 e}$ & $1 / 2$ & $17+18(85)^{\mathrm{a}}$ \\
\hline $\mathrm{NH}_{2} \mathrm{CH}_{2} \mathrm{CH}_{2} \mathrm{CH}_{2} \mathrm{NHCH}_{2} \mathrm{CH}_{2} \mathrm{NHCH}_{2} \mathrm{CH}_{2} \mathrm{CH}_{2} \mathrm{NH}_{2} \mathbf{1 f}$ & $1 / 2$ & $19(88)$ \\
\hline $\mathrm{NH}_{2} \mathrm{CH}_{2} \mathrm{CH}_{2} \mathrm{CH}_{2} \mathrm{~N}(\mathrm{Me}) \mathrm{CH}_{2} \mathrm{CH}_{2} \mathrm{CH}_{2} \mathrm{NH}_{2} \mathbf{~} \mathbf{g}$ & $1 / 2$ & $20(83)$ \\
\hline
\end{tabular}

${ }^{\mathrm{a} A}$ 1:1 mixture of two possible regioisomers.

Table 3 Arylation of sterically hindered diamines.

\begin{tabular}{llcc}
\hline Diamine & \multicolumn{1}{c}{ Aryl bromide } & $\begin{array}{c}\mathrm{PdCl}_{2}(\mathrm{dppf}) / \mathrm{dppf}, \\
\text { mol\% }\end{array}$ & $\begin{array}{c}\text { Product } \\
\text { (yield, \%) }\end{array}$ \\
\hline $\mathrm{NH}_{2} \mathrm{CH}_{2} \mathrm{C}(\mathrm{Me})_{2} \mathrm{CH}_{2} \mathrm{NH}_{2} \mathbf{1 h}$ & $p-\mathrm{C}_{6} \mathrm{H}_{5} \mathrm{C}_{6} \mathrm{H}_{4} \mathrm{Br} \mathbf{2 c}$ & $1 / 1$ & $\mathbf{2 1}(75)$ \\
$\mathrm{NH}_{2} \mathrm{CH}_{2} \mathrm{CH}(\mathrm{Me}) \mathrm{NH}_{2} \mathbf{1 i}$ & $p-\mathrm{C}_{6} \mathrm{H}_{5} \mathrm{C}_{6} \mathrm{H}_{4} \mathrm{Br} \mathbf{2 c}$ & $2 / 3$ & $\mathbf{2 2}(42)$ \\
$\mathrm{NH}_{2} \mathrm{CH}_{2} \mathrm{CH}(\mathrm{Me}) \mathrm{NH}_{2} \mathbf{1 i}$ & 1-bromonaphthalene 2l & $2 / 3$ & $\mathbf{2 3}(64)$ \\
$\mathrm{NH}_{2} \mathrm{CH}_{2} \mathrm{C}(\mathrm{Me})_{2} \mathrm{NH}_{2} \mathbf{1 j}$ & $p-\mathrm{C}_{6} \mathrm{H}_{5} \mathrm{C}_{6} \mathrm{H}_{4} \mathrm{Br} \mathbf{2 c}$ & $2 / 3$ & $\mathbf{2 4}(84)$ \\
1,2-diaminocyclohexane 1k & 1-bromonaphthalene 2l & $1 / 2$ & $\mathbf{2 5}(42)$ \\
\hline
\end{tabular}<smiles>[R]C(N)C(C)(C)C([R])(N)C([R])(C)C([Y])(N)C([R])N</smiles>

1h-k

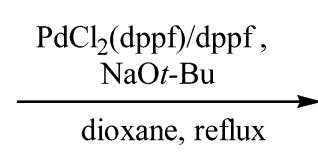

2c, 21<smiles>[R]C(N[Al])C(C)(C)C([R])(N)C([R])([R])N</smiles>

21-25

Scheme 3

$$
\begin{aligned}
& \mathrm{n}=0,1 ; \mathrm{R}^{1}=\mathrm{H}, \mathrm{Me} \\
& \mathrm{R}^{2}=\mathrm{H}, \mathrm{Me} ; \mathrm{R}^{3}=\mathrm{H}, \mathrm{Me}
\end{aligned}
$$




\section{Monoamination of dihaloarenes}

The reaction of polyamines $\mathbf{1 a}, \mathbf{b}, \mathbf{d}, \mathbf{f}$ with dihalobenzenes $\mathbf{2 n}-\mathbf{u}$ was conducted using $\operatorname{Pd}(\mathrm{dba})_{2}(2-4$ mol\%) with dppf (2-6 mol\%) and with polyamine to arylhalide ratio 3:1 [13]. This process afforded corresponding halosubstituted aminobenzenes 26-37 in good yields (Scheme 4, Table 4).<smiles>CC(CC(C)(C)N)NC(C)CC(C)(C)N</smiles>

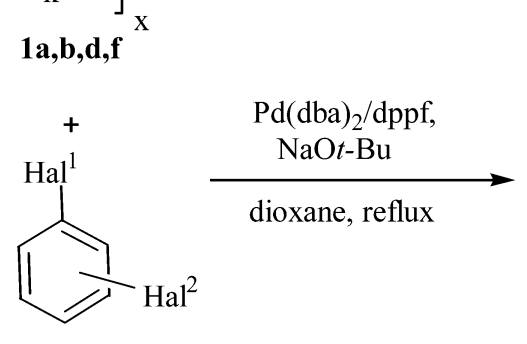

Scheme 4

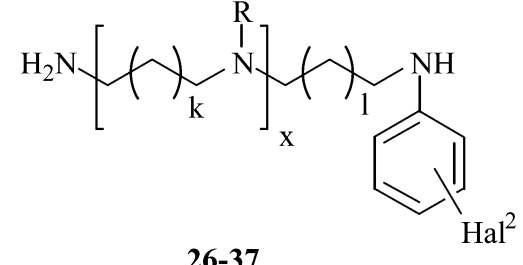

$\mathrm{k}=0,1 ; 1=0,1 ; \mathrm{x}=0,1,2$

Table 4 Monoamination of dihalobenzenes.

\begin{tabular}{|c|c|c|c|}
\hline Amine & Aryl bromide & $\begin{array}{c}\mathrm{Pd}(\mathrm{dba})_{2} / \mathrm{dppf} \\
\mathrm{mol} \%\end{array}$ & $\begin{array}{l}\text { Product } \\
\text { (yield, \%) }\end{array}$ \\
\hline $\mathrm{NH}_{2} \mathrm{CH}_{2} \mathrm{CH}_{2} \mathrm{CH}_{2} \mathrm{NH}_{2} \mathbf{1 a}$ & $p-\mathrm{BrC}_{6} \mathrm{H}_{4} \mathrm{Br} \mathbf{2 n}$ & $2 / 2$ & $26(93)$ \\
\hline $\mathrm{NH}_{2} \mathrm{CH}_{2} \mathrm{CH}_{2} \mathrm{CH}_{2} \mathrm{NH}_{2} \mathbf{~} \mathbf{a}$ & $m-\mathrm{BrC}_{6} \mathrm{H}_{4} \mathrm{Br} 2 \mathrm{o}$ & $2 / 4$ & $27(93)$ \\
\hline $\mathrm{NH}_{2} \mathrm{CH}_{2} \mathrm{CH}_{2} \mathrm{CH}_{2} \mathrm{NH}_{2} \mathbf{1 a}$ & $o-\mathrm{BrC}_{6} \mathrm{H}_{4} \mathrm{Br} \mathbf{2 p}$ & $2 / 3$ & $28(71)$ \\
\hline $\mathrm{NH}_{2} \mathrm{CH}_{2} \mathrm{CH}_{2} \mathrm{CH}_{2} \mathrm{NH}_{2} \mathbf{1 a}$ & $p-\mathrm{BrC}_{6} \mathrm{H}_{4} \mathrm{I} \mathbf{2 q}$ & $4 / 6$ & $26(72)$ \\
\hline $\mathrm{NH}_{2} \mathrm{CH}_{2} \mathrm{CH}_{2} \mathrm{CH}_{2} \mathrm{NH}_{2} \mathbf{1 a}$ & $p-\mathrm{IC}_{6} \mathrm{H}_{4} \mathrm{I} \mathbf{2 r}$ & $4 / 6$ & $29(57)$ \\
\hline $\mathrm{NH}_{2} \mathrm{CH}_{2} \mathrm{CH}_{2} \mathrm{CH}_{2} \mathrm{NH}_{2} \mathbf{~} \mathbf{a}$ & $o-\mathrm{ClC}_{6} \mathrm{H}_{4} \mathrm{Br} 2 \mathrm{~s}$ & $4 / 6$ & $30(75)$ \\
\hline $\mathrm{NH}_{2} \mathrm{CH}_{2} \mathrm{CH}_{2} \mathrm{CH}_{2} \mathrm{NH}_{2} \mathbf{1 a}$ & 2,4-dibromonitrobenzene $\mathbf{2 t}$ & $2 / 3$ & $31(79)$ \\
\hline $\mathrm{NH}_{2} \mathrm{CH}_{2} \mathrm{CH}_{2} \mathrm{NH}_{2} \mathbf{1 b}$ & $p-\mathrm{BrC}_{6} \mathrm{H}_{4} \mathrm{Br} \mathbf{2 n}$ & $2 / 4$ & $32(45)$ \\
\hline $\mathrm{NH}_{2}\left(\mathrm{CH}_{2}\right)_{3} \mathrm{NH}\left(\mathrm{CH}_{2}\right)_{3} \mathrm{NH}_{2} \mathbf{1 d}$ & $p-\mathrm{BrC}_{6} \mathrm{H}_{4} \mathrm{Br} \mathbf{2 n}$ & $2 / 4$ & $33(95)$ \\
\hline $\mathrm{NH}_{2}\left(\mathrm{CH}_{2}\right)_{3} \mathrm{NH}\left(\mathrm{CH}_{2}\right)_{3} \mathrm{NH}_{2} \mathbf{1 d}$ & $m-\mathrm{BrC}_{6} \mathrm{H}_{4} \mathrm{Br} \mathbf{2 o}$ & $2 / 4$ & $34(93)$ \\
\hline $\mathrm{NH}_{2}\left(\mathrm{CH}_{2}\right)_{3} \mathrm{NH}\left(\mathrm{CH}_{2}\right)_{3} \mathrm{NH}_{2} \mathbf{1 d}$ & $o-\mathrm{BrC}_{6} \mathrm{H}_{4} \mathrm{Br} \mathbf{2 p}$ & $2 / 4$ & 35 (74) \\
\hline $\mathrm{NH}_{2}\left(\mathrm{CH}_{2}\right)_{3} \mathrm{NH}\left(\mathrm{CH}_{2}\right)_{3} \mathrm{NH}_{2} \mathbf{1 d}$ & $p-\mathrm{IC}_{6} \mathrm{H}_{4} \mathrm{I}_{2} \mathbf{2} \mathbf{r}$ & $4 / 6$ & $36(48)$ \\
\hline $\mathrm{NH}_{2}\left(\mathrm{CH}_{2}\right)_{3} \mathrm{NH}\left(\mathrm{CH}_{2}\right)_{2} \mathrm{NH}\left(\mathrm{CH}_{2}\right)_{3} \mathrm{NH}_{2}$ 1f & 2,6-dichlorobromobenzene $\mathbf{2 u}$ & $4 / 6$ & $37(85)$ \\
\hline
\end{tabular}

The side process of the reduction of the second halogen atom was unimportant, bromine atom was substituted in the presence of chlorine atom, and iodine atom was substituted in the presence of bromine atom. $P$-Diiodobenzene $\mathbf{2 r}$ was less active than $p$-bromoiodobenzene $\mathbf{2 q}$. Like in previous experiments, only primary amino groups of the triamine $\mathbf{1 d}$ and the tetraamine $\mathbf{1 f}$ were selectively arylated.

\section{Polyarylation of polyamines}

We studied the arylation of di- and triamines $\mathbf{1 a}, \mathbf{b}, \mathbf{d}$ with aryl bromides to find out the conditions of their polyarylation. When running the reactions with equimolar amounts of starting compounds in the presence of $\mathrm{Pd}(\mathrm{dba})_{2} / \mathrm{dppf}$ catalytic system (1-2 mol\% Pd, 2-4 mol\% dppf), generally the mixtures of $\mathrm{N}$-arylated and $\mathrm{N}, \mathrm{N}^{\prime}$-diarylated polyamines were obtained in the case of less active $p$-bromobiphenyl, while more active 1-bromonaphthalene provided selective formation of monoarylated species. When using 2:1 ratio of 1-bromonaphthalene to polyamine, corresponding N,N-diarylated compounds $\mathbf{3 8 , 3 9}$ were obtained in high yields. To achieve good yields of 40-42 with 1-bromonaphthalene BINAP instead 
of dppf should be used. Greater excess of aryl bromides and generous loadings of the catalyst (4 mol\% $\mathrm{Pd}, 8 \mathrm{~mol} \% \mathrm{BINAP}$ ) led to exhaustive polyarylation of di- and triamines and excellent yields of tetraand pentaaryl derivatives 43-46. In these cases, arylation of secondary amino groups in polyamines was observed for the first time (Table 5, Scheme 5).

Table 5 Polyarylation of polyamines.

\begin{tabular}{|c|c|c|c|}
\hline Amine & Aryl bromide & $\begin{array}{l}\mathrm{Pd}(\mathrm{dba})_{2} / \mathrm{dppf} \text { or } \\
\text { BINAP, mol\% }\end{array}$ & $\begin{array}{l}\text { Product } \\
\text { (yield, \%) }\end{array}$ \\
\hline $\mathrm{NH}_{2} \mathrm{CH}_{2} \mathrm{CH}_{2} \mathrm{CH}_{2} \mathrm{NH}_{2} \mathbf{1 a}$ & 1-bromonaphthalene $\mathbf{2 l}$ & $1 / 2$ (dppf) & $38(88) \mathrm{R}=\mathrm{H}$ \\
\hline $\mathrm{NH}_{2}\left(\mathrm{CH}_{2}\right)_{3} \mathrm{NH}\left(\mathrm{CH}_{2}\right)_{3} \mathrm{NH}_{2} \mathbf{1 d}$ & 1-bromonaphthalene $\mathbf{2 l}$ & $1 / 2(\mathrm{dppf})$ & 39 (92) $\mathrm{R}=\mathrm{H}$ \\
\hline $\mathrm{NH}_{2} \mathrm{CH}_{2} \mathrm{CH}_{2} \mathrm{CH}_{2} \mathrm{NH}_{2} \mathbf{1 a}$ & $p-\mathrm{C}_{6} \mathrm{H}_{5} \mathrm{C}_{6} \mathrm{H}_{4} \mathrm{Br} \mathbf{2 c}$ & $2 / 4$ (dppf) & 40 (36) $\mathrm{R}=\mathrm{H}$ \\
\hline $\mathrm{NH}_{2} \mathrm{CH}_{2} \mathrm{CH}_{2} \mathrm{CH}_{2} \mathrm{NH}_{2} \mathbf{1 a}$ & $p-\mathrm{C}_{6} \mathrm{H}_{5} \mathrm{C}_{6} \mathrm{H}_{4} \mathrm{Br} \mathbf{2 c}$ & 2/4 (BINAP) & $40(85) \mathrm{R}=\mathrm{H}$ \\
\hline $\mathrm{NH}_{2} \mathrm{CH}_{2} \mathrm{CH}_{2} \mathrm{NH}_{2} \mathbf{1 b}$ & $p-\mathrm{C}_{6} \mathrm{H}_{5} \mathrm{C}_{6} \mathrm{H}_{4} \mathrm{Br} 2 \mathrm{c}$ & 2/4 (BINAP) & $41(75) \mathrm{R}=\mathrm{H}$ \\
\hline $\mathrm{NH}_{2}\left(\mathrm{CH}_{2}\right)_{3} \mathrm{NH}\left(\mathrm{CH}_{2}\right)_{3} \mathrm{NH}_{2} \mathbf{1 d}$ & $p-\mathrm{C}_{6} \mathrm{H}_{5} \mathrm{C}_{6} \mathrm{H}_{4} \mathrm{Br} \mathbf{2 c}$ & 2/4 (BINAP) & 42 (89) $\mathrm{R}=\mathrm{H}$ \\
\hline $\mathrm{NH}_{2} \mathrm{CH}_{2} \mathrm{CH}(\mathrm{Me}) \mathrm{NH}_{2} \mathbf{1 i}$ & 1-bromonaphthalene $\mathbf{2 l}$ & 6/9 (dppf) & $43(40) \mathrm{R}=\mathrm{H}$ \\
\hline $\mathrm{NH}_{2} \mathrm{CH}_{2} \mathrm{CH}_{2} \mathrm{CH}_{2} \mathrm{NH}_{2} \mathbf{1 a}$ & $p-\mathrm{C}_{6} \mathrm{H}_{5} \mathrm{C}_{6} \mathrm{H}_{4} \mathrm{Br} \mathbf{2 c}$ & 4/8 (BINAP) & $44(95) \mathrm{R}=\mathrm{Ar}$ \\
\hline $\mathrm{NH}_{2} \mathrm{CH}_{2} \mathrm{CH}_{2} \mathrm{NH}_{2} \mathbf{1 b}$ & $p-\mathrm{C}_{6} \mathrm{H}_{5} \mathrm{C}_{6} \mathrm{H}_{4} \mathrm{Br} \mathbf{2 c}$ & 4/8 (BINAP) & $45(95) \mathrm{R}=\mathrm{Ar}$ \\
\hline $\mathrm{NH}_{2}\left(\mathrm{CH}_{2}\right)_{3} \mathrm{NH}\left(\mathrm{CH}_{2}\right)_{3} \mathrm{NH}_{2}$ 1d & $p-\mathrm{C}_{6} \mathrm{H}_{5} \mathrm{C}_{6} \mathrm{H}_{4} \mathrm{Br} \mathbf{2 c}$ & 8/16 (BINAP) & 46 (89) $\mathrm{R}=\mathrm{Ar}$ \\
\hline
\end{tabular}<smiles>[Y]C(CC(C)(C)CN)NCCCC(C)N</smiles>

$\operatorname{Pd}(d b a)_{2}$,
dppf or BINAP,

$\mathbf{1 a , b , d}$

$+$

$\mathrm{ArBr}$

2c, 1

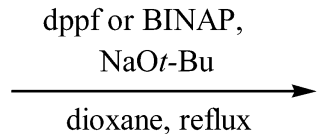

dioxane, reflux<smiles>[R]N(CCCC)CC(C)(C)CC([Y])(CC)N([R])[Al]</smiles>

38-46

Scheme 5

$$
\begin{aligned}
& \mathrm{R}=\mathrm{H}, \mathrm{Ar} \\
& \mathrm{n}=0,1 ; \mathrm{x}=0,1
\end{aligned}
$$

\section{FORMATION OF MACROCYCLES BY INTRAMOLECULAR CATALYTIC AMINATION REACTIONS}

\section{Amination of dihalo- and trihalobenzenes}

To synthesize nitrogen-containing analogs of benzocrown ethers, we tried first the simplest $o$-dibromobenzene $\mathbf{2 p}$ as a substrate in the reaction with tetraamine $\mathbf{1 f}$ [14]. The reaction of equimolar amounts of $2 \mathbf{p}$ and $\mathbf{1 f}$ was run in the presence of $8 \mathrm{~mol} \% \mathrm{Pd}(\mathrm{dba})_{2}$ and $9 \mathrm{~mol} \%$ BINAP, in a diluted solution of boiling dioxane $(\mathrm{c}=0.01 \mathrm{M})$ to avoid possible formation of oligomers, $\mathrm{NaO} t \mathrm{Bu}$ (1.5 equiv) was employed as a base (Scheme 6).

However, the reaction was not enough successful as the desired benzocyclam $\mathbf{4 7}$ was formed in a low $12 \%$ yield together with a linear product of tetraamine diarylation $48(16 \%)$. This can be easily explained by the fact that upon the amination of the first bromine atom, the substitution of the second bromine atom is disfavored by a strong mesomeric donor influence of the amino group in the intermediate $o$-aminobromobenzene, which readily reacts with the second molecule of $o$-dibromobenzene to form 48 . 


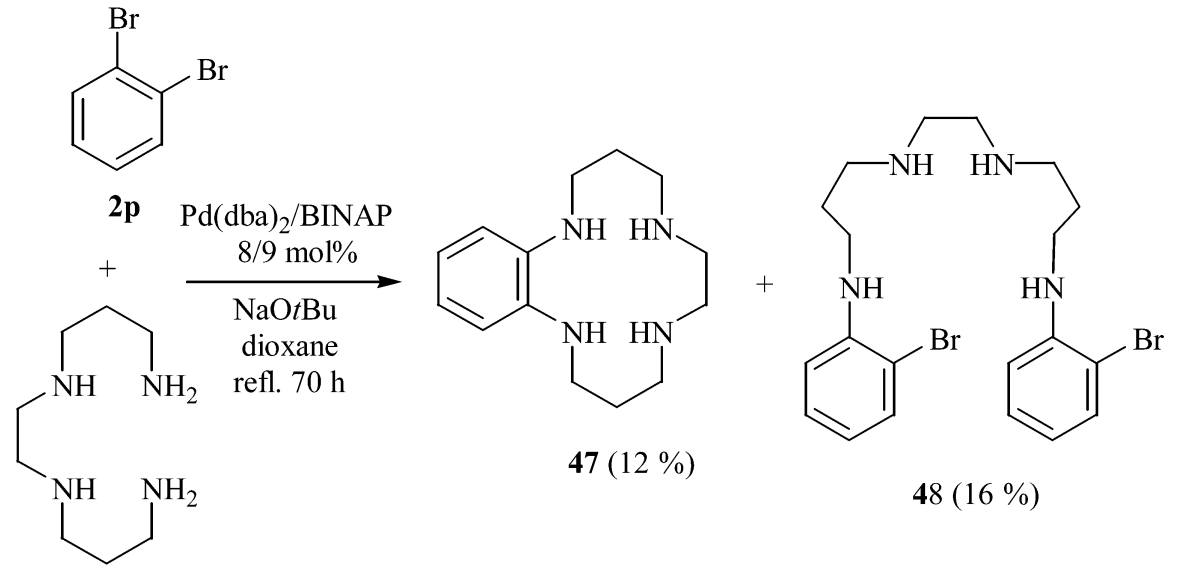

Scheme 6

1f

In order to promote the introduction of the second amino group in the ortho-position to the first amino group, we decided to use 2,6-dichlorobromobenzene $\mathbf{2 u}$ in which the electron-withdrawing influence of the second chlorine atom will to some extent decrease the unfavorable influence of the first introduced amino group. The same reaction conditions were used in the amination of $2 \mathbf{u}$ by a variety of polyamines 1f,l-o (Table 6, Scheme 7).

Table 6 Amination of 2,6-dichlorobromobenzene $2 \mathbf{u}$.

\begin{tabular}{lllllc}
\hline Amine & 1 & $\mathrm{~m}$ & $\mathrm{n}$ & $\mathrm{x}$ & $\begin{array}{c}\text { Product } \\
\text { (yield, \%) }\end{array}$ \\
\hline $\mathrm{NH}_{2} \mathrm{CH}_{2} \mathrm{CH}_{2} \mathrm{CH}_{2} \mathrm{NHCH}_{2} \mathrm{CH}_{2} \mathrm{NHCH}_{2} \mathrm{CH}_{2} \mathrm{CH}_{2} \mathrm{NH}_{2} \mathbf{1 f}$ & 1 & 0 & 1 & 1 & $\mathbf{4 9}(47)$ \\
$\mathrm{NH}_{2} \mathrm{CH}_{2} \mathrm{CH}_{2} \mathrm{NHCH}_{2} \mathrm{CH}_{2} \mathrm{NHCH}_{2} \mathrm{CH}_{2} \mathrm{NH}_{2} \mathbf{1 1}$ & 0 & 0 & 0 & 1 & $\mathbf{5 0}(27)$ \\
$\mathrm{NH}_{2} \mathrm{CH}_{2} \mathrm{CH}_{2} \mathrm{NHCH}_{2} \mathrm{CH}_{2} \mathrm{CH}_{2} \mathrm{NHCH}_{2} \mathrm{CH}_{2} \mathrm{NH}_{2} \mathbf{1 m}$ & 0 & 1 & 0 & 1 & $\mathbf{5 1}(17)$ \\
$\mathrm{NH}_{2} \mathrm{CH}_{2} \mathrm{CH}_{2} \mathrm{NHCH}_{2} \mathrm{CH}_{2} \mathrm{NHCH}_{2} \mathrm{CH}_{2} \mathrm{NHCH}_{2} \mathrm{CH}_{2} \mathrm{NH}_{2} \mathbf{1 n}$ & 0 & 0 & 0 & 2 & $\mathbf{5 2}(12)$ \\
$\mathrm{NH}_{2} \mathrm{CH}_{2} \mathrm{CH}_{2} \mathrm{NHCH}_{2} \mathrm{CH}_{2} \mathrm{NHCH}_{2} \mathrm{CH}_{2} \mathrm{NHCH}_{2} \mathrm{CH}_{2} \mathrm{NHCH}_{2} \mathrm{CH}_{2} \mathrm{NH}_{2} \mathbf{1 o}$ & 0 & 0 & 0 & 3 & $\mathbf{5 3}(10)$ \\
$\mathrm{NH}_{2} \mathrm{CH}_{2} \mathrm{CH}_{2} \mathrm{NHCH}_{2} \mathrm{CH}_{2} \mathrm{NHCH}_{2} \mathrm{CH}_{2} \mathrm{NHCH}_{2} \mathrm{CH}_{2} \mathrm{NH}_{2} \mathbf{1 n} \mathrm{nH}_{2}$ & 0 & 0 & 0 & 2 & $\mathbf{5 4}(17)$ \\
$\mathrm{NH}_{2} \mathrm{CH}_{2} \mathrm{CH}_{2} \mathrm{NHCH}_{2} \mathrm{CH}_{2} \mathrm{NHCH}_{2} \mathrm{CH}_{2} \mathrm{NHCH}_{2} \mathrm{CH}_{2} \mathrm{NHCH}_{2} \mathrm{CH}_{2} \mathrm{NH}_{2} \mathbf{1 o}$ & 0 & 0 & 0 & 3 & $\mathbf{5 5}(21)$ \\
\hline
\end{tabular}

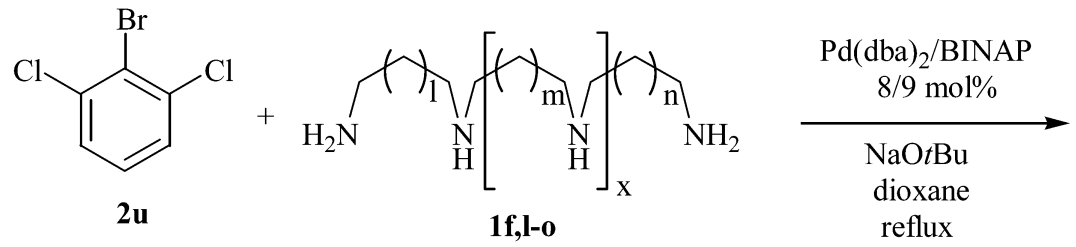

Scheme 7

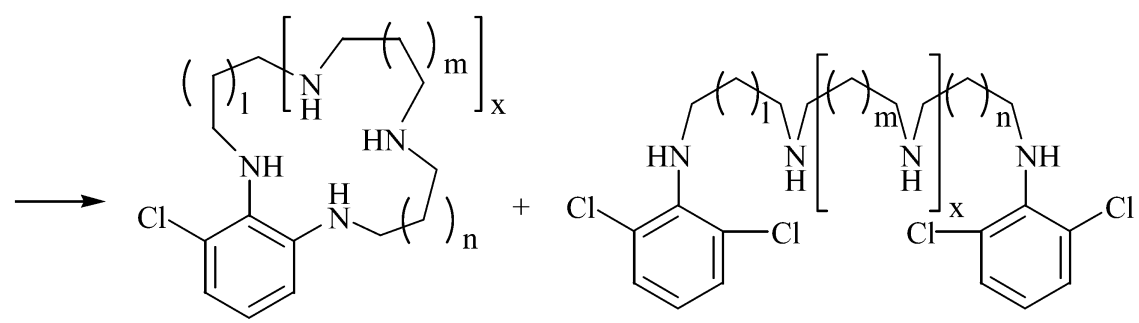

54,55

(C) 2004 IUPAC, Pure and Applied Chemistry 76, 1605-1619 
We have also investigated the reaction of $m$-dibromobenzene 20 in the reaction with tetraamine $1 \mathbf{m}$ to obtain the benzo derivative of cyclam with an unusual configuration of the cycle. The reaction was run under the same conditions to produce the desired product $\mathbf{5 6}$ in $39 \%$ yield (Scheme 8).

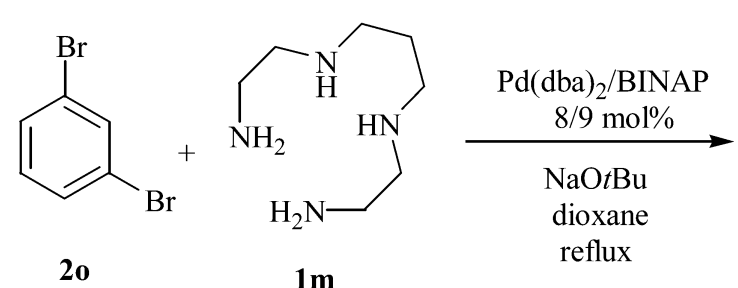<smiles></smiles>

$56(39 \%)$

Scheme 8

\section{Formation of polyazamacrocycles derived from 1,8-dichloroanthracene and 1,8-dichloroanthraquinone}

Macrocyclic compounds containing anthracene and anthraquinone moieties were synthesized by the reaction of equimolar amounts of linear polyamines 1f,l-p with anthracene 57 and anthraquinone $\mathbf{5 8}$ [15]. The $\mathrm{Pd}(\mathrm{dba})_{2} / \mathrm{BINAP}$ catalytic system (4-8 mol\% Pd, 4.5-9 mol\% BINAP) was utilized with $\mathrm{NaO} t \mathrm{Bu}$ base in the case of dichloroanthracene and $\mathrm{Cs}_{2} \mathrm{CO}_{3}$ in the reactions with dichloroanthraquinone. Diluted solutions of the reagents in dioxane were used $(0.017-0.025 \mathrm{M})$ to avoid undesirable formation of oligomers (Table 7, Scheme 9).

Table 7 Amination of 1,8-dichloroanthracene $\mathbf{5 7}$ and 1,8-dichloroanthraquinone $\mathbf{5 8 .}$

\begin{tabular}{lllllcc}
\hline $\begin{array}{l}\text { Aryl } \\
\text { dichloride }\end{array}$ & \multicolumn{1}{c}{ Amine } & 1 & $\mathrm{~m}$ & $\mathrm{n}$ & $\begin{array}{c}\text { Product } \\
\text { (yield, \%) }\end{array}$ \\
\hline $\mathbf{5 7}$ & $\mathrm{NH}_{2} \mathrm{CH}_{2} \mathrm{CH}_{2} \mathrm{CH}_{2} \mathrm{NHCH}_{2} \mathrm{CH}_{2} \mathrm{NHCH}_{2} \mathrm{CH}_{2} \mathrm{CH}_{2} \mathrm{NH}_{2} \mathbf{1 f}$ & 1 & 0 & 1 & 1 & $\mathbf{5 9}(24)$ \\
$\mathbf{5 7}$ & $\mathrm{NH}_{2} \mathrm{CH}_{2} \mathrm{CH}_{2} \mathrm{NHCH}_{2} \mathrm{CH}_{2} \mathrm{NHCH}_{2} \mathrm{CH}_{2} \mathrm{NH}_{2} \mathbf{1 l}$ & 0 & 0 & 0 & 1 & $\mathbf{6 0}(33)$ \\
$\mathbf{5 7}$ & $\mathrm{NH}_{2} \mathrm{CH}_{2} \mathrm{CH}_{2} \mathrm{NHCH}_{2} \mathrm{CH}_{2} \mathrm{CH}_{2} \mathrm{NHCH}_{2} \mathrm{CH}_{2} \mathrm{NH}_{2} \mathbf{1 m}$ & 0 & 1 & 0 & 1 & $\mathbf{6 1}(36)$ \\
$\mathbf{5 7}$ & $\mathrm{NH}_{2} \mathrm{CH}_{2} \mathrm{CH}_{2} \mathrm{NHCH}_{2} \mathrm{CH}_{2} \mathrm{NHCH}_{2} \mathrm{CH}_{2} \mathrm{NHCH}_{2} \mathrm{CH}_{2} \mathrm{NH}_{2} \mathbf{1 n}$ & 0 & 0 & 0 & 2 & $\mathbf{6 2}(26)$ \\
$\mathbf{5 7}$ & $\mathrm{NH}_{2} \mathrm{CH}_{2} \mathrm{CH}_{2} \mathrm{NHCH}_{2} \mathrm{CH}_{2} \mathrm{NHCH}_{2} \mathrm{CH}_{2} \mathrm{NHCH}_{2} \mathrm{CH}_{2} \mathrm{NHCH}_{2} \mathrm{CH}_{2} \mathrm{NH}_{2} \mathbf{1 o}$ & 0 & 0 & 0 & 3 & $\mathbf{6 3}(22)$ \\
$\mathbf{5 7}$ & $\mathrm{NH}_{2} \mathrm{CH}_{2} \mathrm{CH}_{2} \mathrm{CH}_{2} \mathrm{NHCH}_{2} \mathrm{CH}_{2} \mathrm{CH}_{2} \mathrm{NHCH}_{2} \mathrm{CH}_{2} \mathrm{CH}_{2} \mathrm{NH}_{2} \mathbf{1 p}$ & 1 & 1 & 1 & 1 & $\mathbf{6 4}(21)$ \\
$\mathbf{5 8}$ & $\mathrm{NH}_{2} \mathrm{CH}_{2} \mathrm{CH}_{2} \mathrm{CH}_{2} \mathrm{NHCH}_{2} \mathrm{CH}_{2} \mathrm{NH}_{2} \mathrm{CH}_{2} \mathrm{CH}_{2} \mathrm{NH}_{2} \mathbf{1 f}$ & 1 & 0 & 1 & 1 & $\mathbf{6 5}(25)$ \\
$\mathbf{5 8}$ & $\mathrm{NH}_{2} \mathrm{CH}_{2} \mathrm{CH}_{2} \mathrm{NHCH}_{2} \mathrm{CH}_{2} \mathrm{NHCH}_{2} \mathrm{CH}_{2} \mathrm{NH}_{2} \mathbf{1 l}_{2}$ & 0 & 0 & 0 & 1 & $\mathbf{6 6}(14)$ \\
$\mathbf{5 8}$ & $\mathrm{NH}_{2} \mathrm{CH}_{2} \mathrm{CH}_{2} \mathrm{NHCH}_{2} \mathrm{CH}_{2} \mathrm{CH}_{2} \mathrm{NHCH}_{2} \mathrm{CH}_{2} \mathrm{NH}_{2} \mathbf{1 m}$ & 0 & 1 & 0 & 1 & $\mathbf{6 7}(19)$ \\
$\mathbf{5 8}$ & $\mathrm{NH}_{2} \mathrm{CH}_{2} \mathrm{CH}_{2} \mathrm{NHCH}_{2} \mathrm{CH}_{2} \mathrm{NHCH}_{2} \mathrm{CH}_{2} \mathrm{NHCH}_{2} \mathrm{CH}_{2} \mathrm{NH}_{2} \mathbf{1 n}$ & 0 & 0 & 0 & 2 & $\mathbf{6 8}(27)$ \\
$\mathbf{5 8}$ & $\mathrm{NH}_{2} \mathrm{CH}_{2} \mathrm{CH}_{2} \mathrm{NHCH}_{2} \mathrm{CH}_{2} \mathrm{NHCH}_{2} \mathrm{CH}_{2} \mathrm{NHCH}_{2} \mathrm{CH}_{2} \mathrm{NHCH}_{2} \mathrm{CH}_{2} \mathrm{NH}_{2} \mathbf{1 0}$ & 0 & 0 & 0 & 3 & $\mathbf{6 9}(20)$ \\
$\mathbf{5 8}$ & $\mathrm{NH}_{2} \mathrm{CH}_{2} \mathrm{CH}_{2} \mathrm{CH}_{2} \mathrm{NHCH}_{2} \mathrm{CH}_{2} \mathrm{CH}_{2} \mathrm{NHCH}_{2} \mathrm{CH}_{2} \mathrm{CH}_{2} \mathrm{NH}_{2} \mathbf{1 p}$ & 1 & 1 & 1 & $\mathbf{7 0}(10)$ \\
\hline
\end{tabular}


<smiles>Clc1cccc2cc3cccc(Cl)c3cc12</smiles>

$\mathrm{H}_{2} \mathrm{~N}\left(\mathrm{CH}_{2}\right)_{1}\left[\mathrm{NH}\left(\mathrm{CH}_{2}\right)_{\mathrm{m}}\right]_{\mathrm{x}} \mathrm{NH}\left(\mathrm{CH}_{2}\right)_{\mathrm{n}} \mathrm{NH}_{2}$ 1f, l-p

57

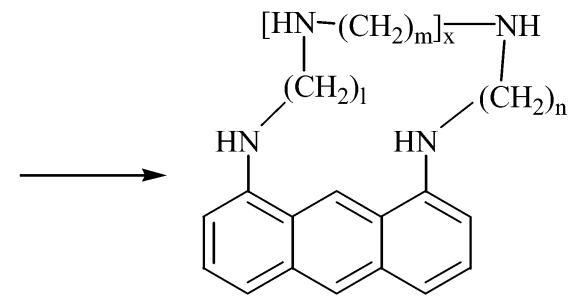

59-64<smiles>O=C1c2cccc(Cl)c2C(=O)c2cccc(Cl)c21</smiles>

$$
\begin{gathered}
\mathrm{H}_{2} \mathrm{~N}\left(\mathrm{CH}_{2}\right)_{1}\left[\mathrm{NH}\left(\mathrm{CH}_{2}\right)_{\mathrm{m}}\right]_{\mathrm{x}} \mathrm{NH}\left(\mathrm{CH}_{2}\right)_{\mathrm{n}_{\mathbf{n}}} \mathrm{NH}_{2} \\
\text { 1f, l-p } \\
\hline
\end{gathered}
$$

$\mathrm{Pd}(\mathrm{dba})_{2} / \mathrm{BINAP}, \quad \mathrm{Cs}_{2} \mathrm{CO}_{3}, \quad$ dioxane

58

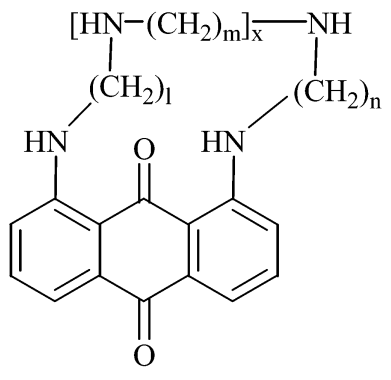

\section{Scheme 9}

65-70

The reduction of chlorine atom generally was not observed in the syntheses of the anthracenebased macrocycles. In contrast, the reactions of dichloroanthraquinone $\mathbf{5 8}$ with polyamines led to a substantial formation of linear compounds: a mixture of 1-amino- and 1-amino-8-chloroanthraquinones with yields up to $30 \%$, as well as the compounds with higher molecular weight and anthraquinone/amine ratio 2:1. While the conversion of $\mathbf{5 7}$ was full, the conversion of $\mathbf{5 8}$ was $90-95 \%$ and some amount was reduced into anthraquinone and 1-chloroanthraquinone. In all mentioned reactions, only primary nitrogen atoms were arylated.

A new family of diazacrown ethers that incorporate anthracene or anthraquinone moiety was synthesized using the same method of Pd-catalyzed amination of 1,8-dichloroanthracene and 1,8-dichloroanthraquinone by employing 3,6-dioxa-1,8-diaminooctane 1q, 4,9-dioxa-1,12-diaminododecane 1r, and 4,7,10-trioxa-1,13-diaminotridecane 1s [16]. The experimental procedure elaborated for the synthesis of tetraazamacrocycles 59-70 was successfully applied to the synthesis of diazacrown ethers 71-76 (Table 8, Scheme 10). 
Table 8 Amination of 1,8-dichloroanthracene 57 and 1,8-dichloroanthraquinone $\mathbf{5 8}$ by di- and trioxadiamines.

\begin{tabular}{llc}
\hline $\begin{array}{l}\text { Aryl } \\
\text { dichloride }\end{array}$ & \multicolumn{1}{c}{ Amine } & $\begin{array}{c}\text { Product } \\
\text { (yield, \%) }\end{array}$ \\
\hline $\mathbf{5 7}$ & $\mathrm{NH}_{2} \mathrm{CH}_{2} \mathrm{CH}_{2} \mathrm{OCH}_{2} \mathrm{CH}_{2} \mathrm{OCH}_{2} \mathrm{CH}_{2} \mathrm{NH}_{2} \mathbf{1 q}$ & $\mathbf{7 1}(28)$ \\
$\mathbf{5 7}$ & $\mathrm{NH}_{2} \mathrm{CH}_{2} \mathrm{CH}_{2} \mathrm{CH}_{2} \mathrm{OCH}_{2} \mathrm{CH}_{2} \mathrm{OCH}_{2} \mathrm{CH}_{2} \mathrm{OCH}_{2} \mathrm{CH}_{2} \mathrm{CH}_{2} \mathrm{NH}_{2} \mathbf{1 s}$ & $\mathbf{7 2}(20)$ \\
$\mathbf{5 7}$ & $\mathrm{NH}_{2} \mathrm{CH}_{2} \mathrm{CH}_{2} \mathrm{CH}_{2} \mathrm{OCH}_{2} \mathrm{CH}_{2} \mathrm{CH}_{2} \mathrm{CH}_{2} \mathrm{OCH}_{2} \mathrm{CH}_{2} \mathrm{CH}_{2} \mathrm{NH}_{2} \mathbf{~ r}$ & $\mathbf{7 3}(25)$ \\
$\mathbf{5 8}$ & $\mathrm{NH}_{2} \mathrm{CH}_{2} \mathrm{CH}_{2} \mathrm{OCH}_{2} \mathrm{CH}_{2} \mathrm{OCH}_{2} \mathrm{CH}_{2} \mathrm{NH}_{2} \mathbf{1 q}$ & $\mathbf{7 4}(36)$ \\
$\mathbf{5 8}$ & $\mathrm{NH}_{2} \mathrm{CH}_{2} \mathrm{CH}_{2} \mathrm{CH}_{2} \mathrm{OCH}_{2} \mathrm{CH}_{2} \mathrm{OCH}_{2} \mathrm{CH}_{2} \mathrm{OCH}_{2} \mathrm{CH}_{2} \mathrm{CH}_{2} \mathrm{NH}_{2} \mathbf{1 s}$ & $\mathbf{7 5}(37)$ \\
$\mathbf{5 8}$ & $\mathrm{NH}_{2} \mathrm{CH}_{2} \mathrm{CH}_{2} \mathrm{CH}_{2} \mathrm{OCH}_{2} \mathrm{CH}_{2} \mathrm{CH}_{2} \mathrm{CH}_{2} \mathrm{OCH}_{2} \mathrm{CH}_{2} \mathrm{CH}_{2} \mathrm{NH}_{2} \mathbf{~ q}$ & $\mathbf{7 6}(33)$ \\
\hline
\end{tabular}<smiles>Clc1cccc2cc3cccc(Cl)c3cc12</smiles>

57

or<smiles>O=C1c2cccc(Cl)c2C(=O)c2cccc(Cl)c21</smiles>

58

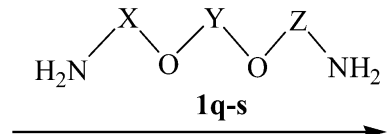

$\mathrm{Pd}(\mathrm{dba})_{2} / \mathrm{BINAP}$

$\mathrm{NaO} t \mathrm{Bu}$ or $\mathrm{Cs}_{2} \mathrm{CO}_{3}$ dioxane

\section{Scheme 10}<smiles>[X]Nc1cccc2cc3cccc(N[Y]([Z])O[X])c3cc12</smiles>

71-73<smiles>[Y]Nc1cccc2c1C(=O)c1c(N[Z]([X])([H])O[Y]O[Z])cccc1C2=O</smiles>

The anthracene-based macrocycles 71-73 were obtained in 20-29\% yields, while the yields of the anthraquinone-based crown ethers 74-76 were even higher (33-37\%). Complete conversion of starting aryl halides was observed in all cases. It should be noted that the main side-products of this reaction were the cyclic dimers and trimers (Fig. 1) which were isolated in relatively high yields (up to 18 and $8 \%$, respectively). Using MALDI-TOF mass spectroscopy, we revealed the formation of the cyclooligomers with higher masses, even cyclodecamers with $M>4200$. The application of more diluted solutions substantially suppressed the formation of cyclic oligomers.
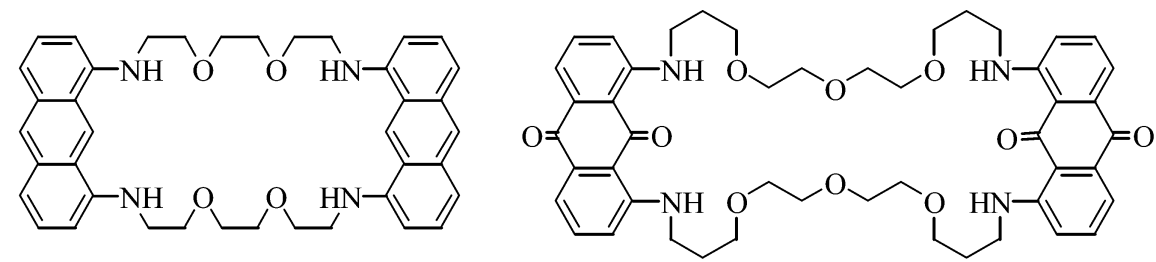

Fig. 1 Examples of cyclodimers. 


\section{Polyazamacrocycles based on 1,5-disubstituted anthracene and anthraquinone}

The same conditions were employed for the synthesis of the macrocycles based on 1,5-disubstituted anthracene. The tetraamines $\mathbf{1 f}, \mathbf{l}, \mathbf{m}, \mathbf{p}$ were tested in this reaction with 1,5-dichloroanthracene $\mathbf{7 7}$. The geometry of the starting compound $\mathbf{7 7}$ implies that the aliphatic chain should be long enough to accomplish cyclization. Tetraamine $\mathbf{1 l}$ was too short to form the desired macrocycle $\mathbf{7 8}$ in a reasonable yield. Only traces of $\mathbf{7 8}$ were detected together with its cyclic dimer isolated in $18 \%$ yield. Much better results were achieved with the tetraamine $\mathbf{1 m}$ whose chain is only by one carbon atom longer than that of $\mathbf{1 1}$. In this case, monomeric cycle $\mathbf{7 9}$ was obtained in $20 \%$ yield. Its cyclic dimer was also isolated in $10 \%$ yield The same reaction was successfully run with tetraamines $\mathbf{1 f}$ and $\mathbf{1 p}$ to produce corresponding macrocycles $\mathbf{8 0}$ and $\mathbf{8 1}$ in 34 and $22 \%$ yields, respectively (Table 9, Scheme 11). These reactions also produced substantial amounts of cyclic oligomers.

Table 9 Amination of 1,5-dichloroanthracene 77.

\begin{tabular}{ll}
\hline Amine & $\begin{array}{c}\text { Product } \\
\text { (yield, \%) }\end{array}$ \\
\hline $\mathrm{NH}_{2} \mathrm{CH}_{2} \mathrm{CH}_{2} \mathrm{CH}_{2} \mathrm{NHCH}_{2} \mathrm{CH}_{2} \mathrm{NHCH}_{2} \mathrm{CH}_{2} \mathrm{CH}_{2} \mathrm{NH}_{2} \mathbf{1 f}$ & $\mathbf{8 0}(34)$ \\
$\mathrm{NH}_{2} \mathrm{CH}_{2} \mathrm{CH}_{2} \mathrm{NHCH}_{2} \mathrm{CH}_{2} \mathrm{NHCH}_{2} \mathrm{CH}_{2} \mathrm{NH}_{2} \mathbf{1 l}$ & $\mathbf{7 8}(0)$ \\
$\mathrm{NH}_{2} \mathrm{CH}_{2} \mathrm{CH}_{2} \mathrm{NHCH}_{2} \mathrm{CH}_{2} \mathrm{CH}_{2} \mathrm{NHCH}_{2} \mathrm{CH}_{2} \mathrm{NH}_{2} \mathbf{1 m}$ & $\mathbf{7 9}(20)$ \\
$\mathrm{NH}_{2} \mathrm{CH}_{2} \mathrm{CH}_{2} \mathrm{CH}_{2} \mathrm{NHCH}_{2} \mathrm{CH}_{2} \mathrm{CH}_{2} \mathrm{NHCH}_{2} \mathrm{CH}_{2} \mathrm{CH}_{2} \mathrm{NH}_{2} \mathbf{1 p}$ & $\mathbf{8 1}(22)$ \\
$\mathrm{NH}_{2} \mathrm{CH}_{2} \mathrm{CH}_{2} \mathrm{OCH}_{2} \mathrm{CH}_{2} \mathrm{OCH}_{2} \mathrm{CH}_{2} \mathrm{NH}_{2} \mathbf{1 q}$ & $\mathbf{8 2}(0)$ \\
$\mathrm{NH}_{2} \mathrm{CH}_{2} \mathrm{CH}_{2} \mathrm{CH}_{2} \mathrm{OCH}_{2} \mathrm{CH}_{2} \mathrm{OCH}_{2} \mathrm{CH}_{2} \mathrm{OCH}_{2} \mathrm{CH}_{2} \mathrm{CH}_{2} \mathrm{NH}_{2} \mathbf{1 s}$ & $\mathbf{8 3}(24)$ \\
$\mathrm{NH}_{2} \mathrm{CH}_{2} \mathrm{CH}_{2} \mathrm{CH}_{2} \mathrm{OCH}_{2} \mathrm{CH}_{2} \mathrm{CH}_{2} \mathrm{CH}_{2} \mathrm{OCH}_{2} \mathrm{CH}_{2} \mathrm{CH}_{2} \mathrm{NH}_{2} \mathbf{1 r}$ & $\mathbf{8 4}(20)$ \\
\hline
\end{tabular}<smiles>Clc1cccc2cc3c(Cl)cccc3cc12</smiles>

77

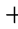<smiles>CCCCCNc1cccc2cc3c(NCCCC)cccc3cc12</smiles>

78-81

Scheme 11

$$
\mathrm{H}_{2} \mathrm{~N}\left(\mathrm{CH}_{2}\right)_{1} \mathrm{NH}\left(\mathrm{CH}_{2}\right)_{\mathrm{m}} \mathrm{NH}\left(\mathrm{CH}_{2}\right)_{n} \mathrm{NH}_{2}
$$

1f,m,p

The reaction of 1,5-dichloroanthracene 77 with dioxadiamine $\mathbf{1 q}$, which possesses the same chain length as $\mathbf{1 1}$, did not lead to the formation of the macrocycle of type $\mathbf{8 2}$, but rather afforded a cyclic dimer and a mixture of linear oligomers. Both reactions with diamines 1r,s resulted in the formation of corresponding oxaazacyclophanes $\mathbf{8 3 , 8 4}$ in reasonable 24 and $20 \%$ yields (Table 9, Scheme 12). 


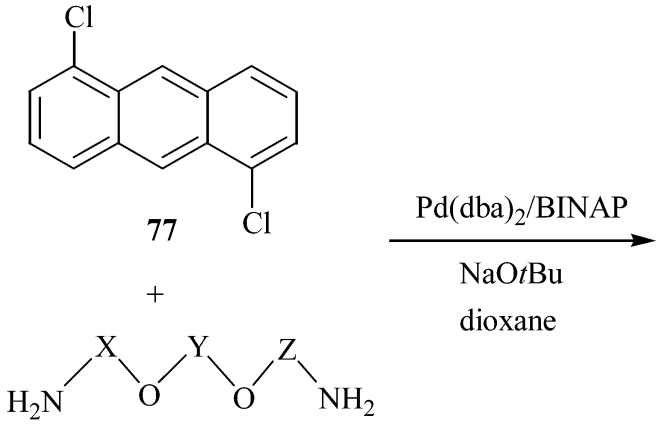

$18 q-s$

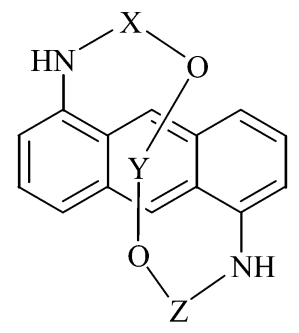

82-84

Scheme 12

The amination of 1,5-dichloroanthraquinone 85 with tetraamines $\mathbf{1 f ,} \mathbf{l}-\mathbf{n}$ and di- and trioxadiamines 1q-s led to substantially different results in comparison with the case of 1,5-dichloroanthracene. Diazacrown ethers $\mathbf{8 6 , 8 7}$ were obtained in good yields provided $\mathrm{Cs}_{2} \mathrm{CO}_{3}$ was employed as a base instead of $\mathrm{NaO} t \mathrm{Bu}$. Similarly, the amination of a shorter dioxadiamine $\mathbf{1 q}$ did not afford the target macrocycle, but rather produced its cyclic dimer in $18 \%$ yield. In the case of the reaction of $\mathbf{1 r}$, the target macrocycle $\mathbf{8 6}$ was obtained in $30 \%$ yield and the cyclic dimer and trimer were isolated in 10 and $6 \%$ yields, respectively. The reaction of $\mathbf{8 5}$ with $\mathbf{1 s}$ furnished macrocycle $\mathbf{8 7}$ in $28 \%$ yield (Table 10, Scheme 13).

Table 10 Amination of 1,5-dichloroanthraquinone $\mathbf{8 5}$.

\begin{tabular}{ll}
\hline Amine & Product (yield, \%) \\
\hline $\mathrm{NH}_{2} \mathrm{CH}_{2} \mathrm{CH}_{2} \mathrm{CH}_{2} \mathrm{OCH}_{2} \mathrm{CH}_{2} \mathrm{OCH}_{2} \mathrm{CH}_{2} \mathrm{OCH}_{2} \mathrm{CH}_{2} \mathrm{CH}_{2} \mathrm{NH}_{2} \mathbf{1 s}$ & $\mathbf{8 6}(30)$ \\
$\mathrm{NH}_{2} \mathrm{CH}_{2} \mathrm{CH}_{2} \mathrm{CH}_{2} \mathrm{OCH}_{2} \mathrm{CH}_{2} \mathrm{CH}_{2} \mathrm{CH}_{2} \mathrm{OCH}_{2} \mathrm{CH}_{2} \mathrm{CH}_{2} \mathrm{NH}_{2} \mathbf{~ 1 r}$ & $\mathbf{8 7}(28)$ \\
$\mathrm{NH}_{2} \mathrm{CH}_{2} \mathrm{CH}_{2} \mathrm{CH}_{2} \mathrm{NHCH}_{2} \mathrm{CH}_{2} \mathrm{NHCH}_{2} \mathrm{CH}_{2} \mathrm{CH}_{2} \mathrm{NH}_{2} \mathbf{1 f}$ & $\mathbf{8 8}(23)+\mathbf{9 2}(10)$ \\
$\mathrm{NH}_{2} \mathrm{CH}_{2} \mathrm{CH}_{2} \mathrm{NHCH}_{2} \mathrm{CH}_{2} \mathrm{NHCH}_{2} \mathrm{CH}_{2} \mathrm{NH}_{2} \mathbf{1 l}$ & $\mathbf{8 9}(28)+\mathbf{9 3}(10)$ \\
$\mathrm{NH}_{2} \mathrm{CH}_{2} \mathrm{CH}_{2} \mathrm{NHCH}_{2} \mathrm{CH}_{2} \mathrm{CH}_{2} \mathrm{NHCH}_{2} \mathrm{CH}_{2} \mathrm{NH}_{2} \mathbf{~} \mathbf{~ m}$ & $\mathbf{9 0}(25)+\mathbf{9 4}(5)$ \\
$\mathrm{NH}_{2} \mathrm{CH}_{2} \mathrm{CH}_{2} \mathrm{CH}_{2} \mathrm{NHCH}_{2} \mathrm{CH}_{2} \mathrm{CH}_{2} \mathrm{NHCH}_{2} \mathrm{CH}_{2} \mathrm{CH}_{2} \mathrm{NH}_{2} \mathbf{1 p}$ & $\mathbf{9 1}(25)+\mathbf{9 5}(26)$ \\
\hline
\end{tabular}<smiles>O=C1c2cccc(Cl)c2C(=O)c2cccc(Cl)c21</smiles>

85

$+$<smiles>[Z][CH]O[Y]O[Y]</smiles>

$1 \mathbf{r , s}$

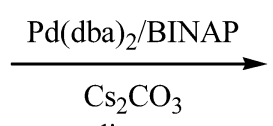

dioxane<smiles>[Z]Nc1cccc(C(=O)OC)c1C(=O)Nc1cccc([X])c1C(=O)OC</smiles>

86, 87

\section{Scheme 13}

Surprisingly, the amination of 1,5-dichloroanthraquinone 85 by tetraamines $\mathbf{1 f}, \mathbf{m}, \mathbf{p}$ did not lead to the formation of corresponding tetraazamacrocycles. Only 1-amino-5-chlorosubstituted anthraquinones 88-91 were built in 16-28\% yields together with bis(aryl)substituted tetraamines 92-95, 
which were formed in 5-26\% yields (Table 10, Scheme 14). The consumption of 1,5-dichloroanthraquinone was not quantitative like in the reactions of 1,8-dichloroanthraquinone with tetraamines, and partial reduction of the chlorine atom was observed. The attempts to promote the formation of
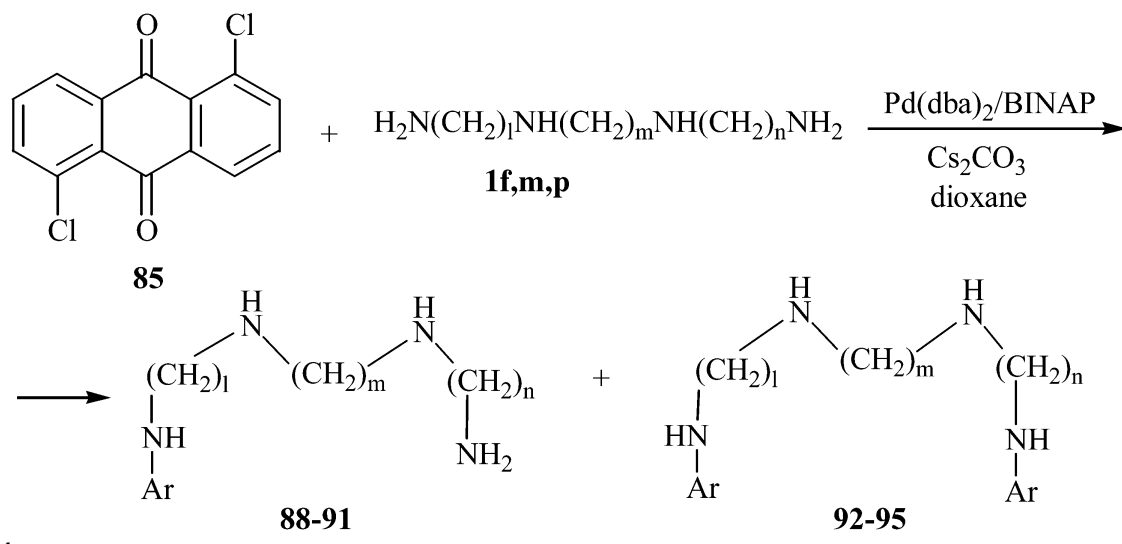

\section{Scheme 14}

macrocycles using double amount of the catalyst, the application of other phosphine ligands were not successful.

\section{ARYLATION OF CYCLIC POLYAMINES AND AZACROWN ETHERS}

\section{Pd-catalyzed arylation of cyclam}

Another approach to N-arylsubstituted polyazamacrocycles comprises direct catalytic arylation of such cyclic polyamines. Due to the fact that secondary cyclic amines are often problematic substrates in the amination reactions we tried various combinations of the catalyst precursor with supporting ligand in the reactions of 1,4,8,11-tetraazacyclotetradecane (cyclam) 96 with arylhalides (chloro-, bromo-, iodosubstituted benzenes, 1-bromonaphthaline, 4-bromobiphenyl, and 9-bromoanthracene). We tested $\mathrm{Pd}(\mathrm{dba})_{2}, \mathrm{Pd}(\mathrm{OAc})_{2}, \mathrm{PdCl}_{2}$, in the combinations with dppf, BINAP, $\mathrm{P} t \mathrm{Bu}_{3}, \mathrm{P}(\mathrm{o}-\mathrm{Tol})_{3}, \mathrm{P}(\mathrm{Cy})_{3}$, but none

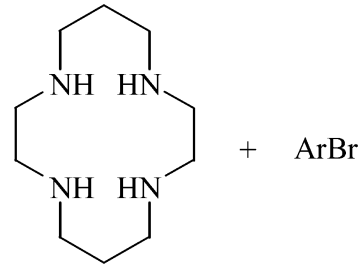

96

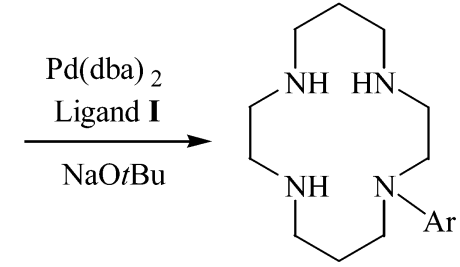

97,98

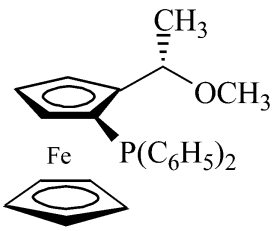

Ligand I

$$
\mathrm{Ar}=\mathrm{p}-\mathrm{C}_{6} \mathrm{H}_{5} \mathrm{C}_{6} \mathrm{H}_{4}(\mathbf{9 7}, 20 \%), p-\mathrm{NCC}_{6} \mathrm{H}_{4}(\mathbf{9 8}, 22 \%)
$$

\section{Scheme 15}

of them proved to be applicable to our purpose. The sole result was full or partial reduction of aryl halides into corresponding hydrocarbons by amines. At last, we managed to run the desirable reaction of cyclam with 4-bromobiphenyl and 4-bromobenzonitrile using PPF-OMe ligand, which afforded $N$-arylated cyclams 97 and 98 in 20-22\% yields (Scheme 15) [17]. 


\section{Synthesis of face-to-face arranged bismacrocyclic systems}

To solve the problem of a simple one-pot synthesis of saturated analog of cofacial porphyrins [18], we chose 1,8-disubstituted anthracene as a spacer. At first, we tried a cheaper catalytic system $\mathrm{Pd}(\mathrm{dba})_{2} / \mathrm{BINAP}$ rather than more expensive PPF-OMe. The reaction of 1,8-dichloroanthracene with cyclam in the presence of $4-8 \mathrm{~mol} \%$ of $\mathrm{Pd}(\mathrm{dba})_{2} / \mathrm{BINAP}$ and $\mathrm{NaO} t \mathrm{Bu}$ led only to anthracene- the product of the reduction of the starting dichloride. The same reaction with less basic 1,4,7,10-tetraazacyclododecane (cyclen) 99 in the presence of $5 \mathrm{~mol} \% \mathrm{Pd}(\mathrm{dba})_{2} / \mathrm{BINAP}$ proved to be more successful, generally yielding a mixture of monocyclen-substituted anthracenes 100 and 101 together with free anthracene (Scheme 16). However, disubstituted anthracene with two cyclen rings was not obtained. In order to attenuate undesirable reduction we thought to apply $\mathrm{N}_{1} \mathrm{~N}^{\prime}, \mathrm{N}^{\prime \prime}$-trisubstituted derivatives of cyclam instead of free cyclam, which contains 4 secondary amino groups capable of participating in the reduction of dichloroanthracene. BINAP and $\mathrm{PBut}_{3}$ were tried as supporting ligands. Reactions of

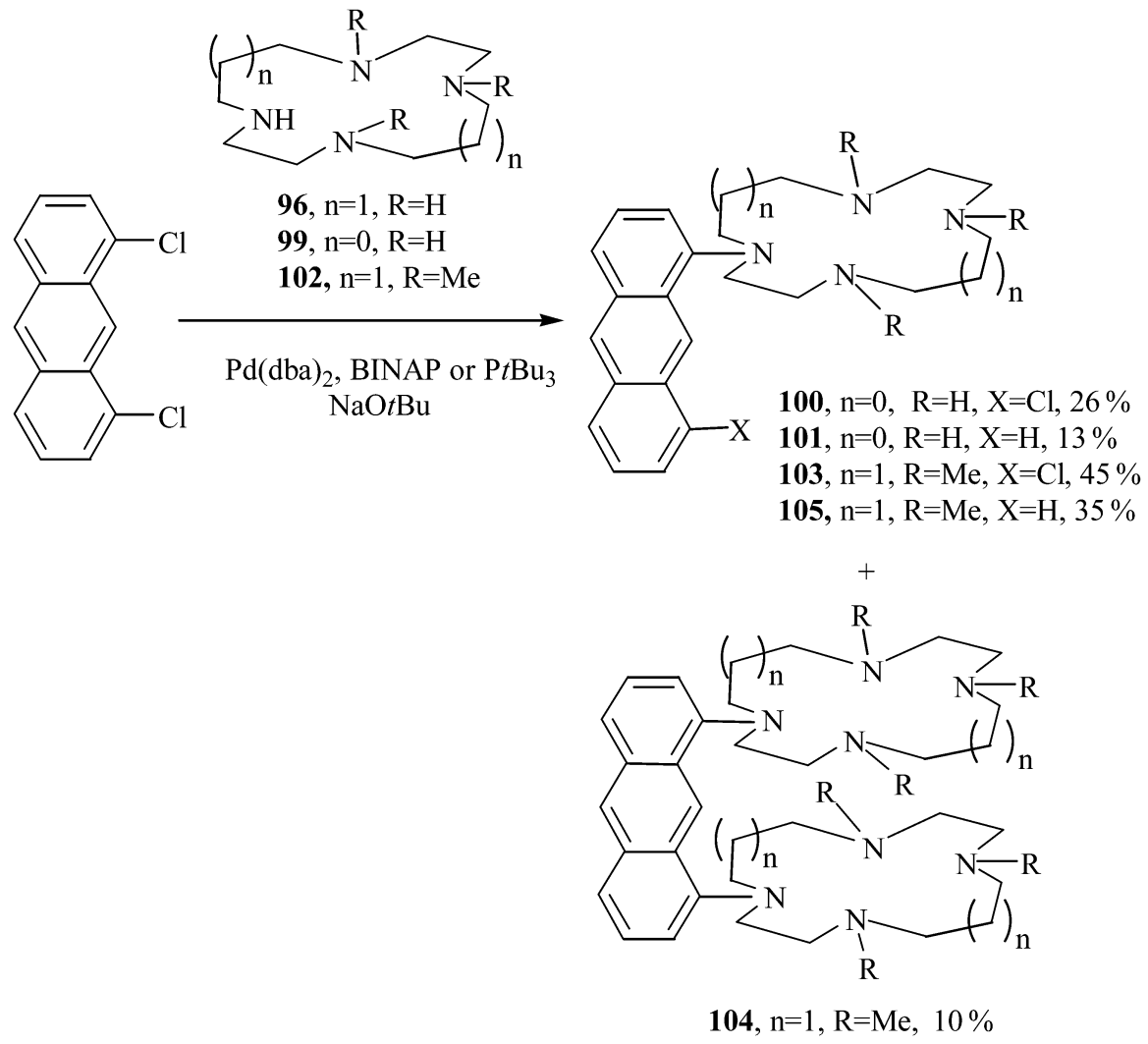

Scheme 16

$N, N^{\prime}, N^{\prime \prime}$-trimethylcyclam 102 promoted by $\mathrm{Pd}$ precursor $\left(\mathrm{Pd}(\mathrm{dba})_{2}, 16 \mathrm{~mol} \%\right)$ with $\mathrm{P} t \mathrm{Bu} \mathrm{u}_{3}$ produced monocyclam derivative of anthracene $\mathbf{1 0 3}$ in $45 \%$ yield but only traces of desirable biscyclam product 104. Further attempts to synthesize it using $\mathrm{Pd}(\mathrm{dba})_{2} / \mathrm{BINAP}$ catalytic system (16 mol\%) resulted in the 

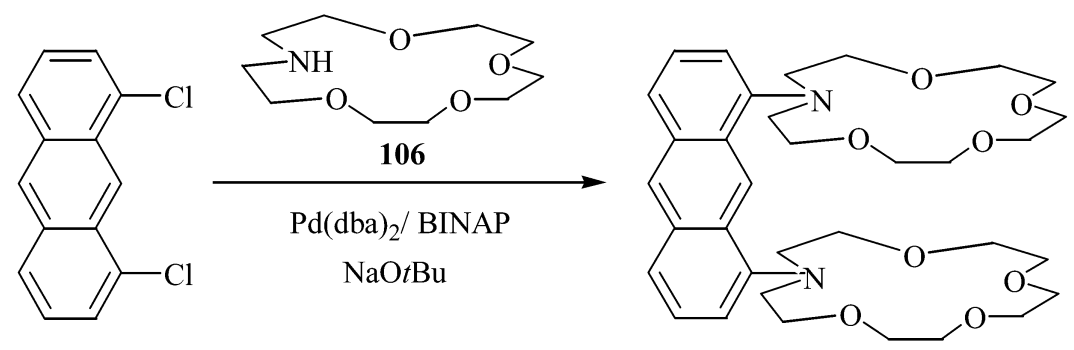

$107(10 \%)$

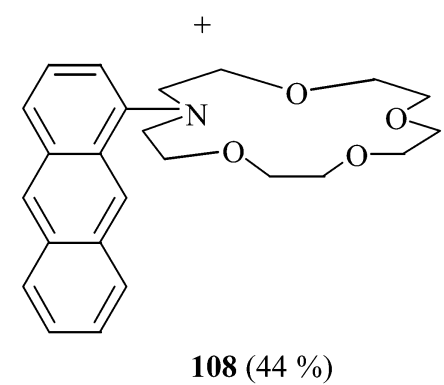

\section{Scheme 17}

formation of the target compound $\mathbf{1 0 4}$ in $10 \%$ yield together with monosubstituted anthracene $\mathbf{1 0 5}$ (35\%) (Scheme 16).

We have also shown the applicability of used methodology to the synthesis of bis(azacrown) substituted anthracene. We investigated the possibility to synthesize a face-to-face ring system employing two azacrown moeities. In our case, 1-aza-15-crown-5 106 was reacted with 1,8-dichloroanthracene in the presence of $\mathrm{Pd}(\mathrm{dba})_{2} / \mathrm{BINAP}$ catalytic system to obtain target biscrown substituted anthracene $\mathbf{1 0 7}$ in $11 \%$ yield along with monosubstituted compound 108 (44\%) (Scheme 17).

In conclusion, the data collected in our research allows us to choose the best catalytic system, which is applicable to the amination reactions of various aryl halides that employ polyamines. This system contains $\mathrm{Pd}(\mathrm{dba})_{2}$ as a catalyst precursor with BINAP ligand, which also proved to be a versatile tool in other amination reactions [19] where monoamines are used. In most cases, Pd(dba) /BINAP catalytic system provides the best results, first of all in intramolecular cyclizations leading to polyazamacrocycles.

\section{ACKNOWLEDGMENTS}

This work was supported in part by RFBR Grant Nos. 02-03-33331 and 03-03-32627.

\section{REFERENCES}

1. J. S. Bradshaw, K. E. Krakowiak, R. M. Izatt. Tetrahedron 48, 4475 (1992).

2. J. S. Bradshaw, K. E. Krakowiak, R. M. Izatt. Aza-Crown Macrocycles, p. 51, John Wiley, New York (1993).

3. L. F. Lindoy. The Chemistry of Macrocyclic Ligand Complexes, Cambridge University Press, Cambridge (1989).

4. J.-M. Lehn. Supramolecular Chemistry: Concepts and Perspectives, VCH, Weinheim (1995).

5. C. Bazzicalupi, A. Bencini, H. Cohen, C. Giorgi, G. Golub, D. Meyerstein, N. Navon, P. Paoletti, B. Valtancoli. J. Chem. Soc., Dalton Trans. 1625 (1998). 
6. J. Arago, A. Bencini, A. Bianchi, E. Garcia-Espana, M. Micheloni, P. Paoletti, J. A. Ramirez, P. Paoli. Inorg. Chem. 30, 1843 (1991).

7. M. A. Bernardo, J. A. Guerrero, E. Garcia-Espana, S. V. Luis, J. M. Llinares, F. Pina, J. A. Ramirez, C. Soriano. J. Chem. Soc., Perkin Trans 22335 (1996).

8. M. A. Bernardo, F. Pina, E. Garcia-Espana, J. Latorre, S. V. Luis, J. M. Llinares, J. A. Ramirez, C. Soriano. Inorg. Chem. 37, 3935 (1998).

9. L. Fourteau, E. Benoist, M. Dartiguenave. Synlett 1, 126 (2001).

10. G. S. Poindexter, D. A. Owens, P. L. Dolan, E. Woo. J. Org. Chem. 57, 6257 (1992).

11. B. H. Yang and S. L. Buchwald. J. Organomet. Chem. 576, 125 (1999).

12. R. Guilard, A. G. Bessmertnykh, I. P. Beletskaya. Tetrahedron Lett. 38, 2287 (1997).

13. R. Guilard, A. G. Bessmertnykh, I. P. Beletskaya. Synlett 1459 (1999).

14. I. P. Beletskaya, A. D. Averin, A. A. Borisenko, F. Denat, R. Guilard. Tetrahedron Lett. 44, 1433 (2003).

15. I. P. Beletskaya, A. D. Averin, A. G. Bessmertnykh, R. Guilard. Tetrahedron Lett. 42, 4983 (2001).

16. I. P. Beletskaya, A. D. Averin, A. G. Bessmertnykh, R. Guilard. Tetrahedron Lett. 42, 4987 (2001).

17. I. P. Beletskaya, A. D. Averin, A. G. Bessmertnykh, F. Denat, R. Guilard. Tetrahedron Lett. 43, 1193 (2002).

18. S. Brandes, C. Gros, F. Denat, P. Pullumbi, R. Guilard. Bull. Soc. Chim. Fr. 133, 65 (1996).

19. J. P. Wolfe and S. L. Buchwald. J. Org. Chem. 65, 1144 (2000). 